\title{
Postgonococcal urethritis: a double-blind study of doxycycline vs placebo
}

\author{
K A Mclean, B A Evans, J M H Lim, B S Azadin
}

\begin{abstract}
The occurrence of postgonococcal urethritis (PGU) was studied in heterosexual men who were randomly assigned to receive either oral doxycycline $100 \mathrm{mg}$ or a placebo capsule twice daily for seven days in addition to routine single dose treatment of urethral gonorrhoea. Of 216 patients suitable for assessment, PGU was diagnosed in 54/104 (51.9\%) in the placebo group and $30 / 112(26.8 \%)$ in the doxycycline group. Symptomatic PGU was uncommon (6.7\% in the placebo group, $2.7 \%$ in the doxycycline group). Persistence of chlamydial infection at first follow-up was associated with the development of PGU in the placebo group (p < 0.05). Although PGU developed in 10/12 (83\%) patients in whom Mycoplasma hominis was isolated on entry to the study, the association was not statistically significant. The development of PGU was not associated with the isolation of Ureaplasma urealyticum. A significant reduction in the incidence of PGU was achieved by the addition of a course of doxycycline $(p<0.001)$, but there remained a considerable proportion of patients in whom the aetiology of PGU was unknown.
\end{abstract}

Postgonococcal urethritis (PGU) occurs after single dose treatment of urethral gonorrhoea in heterosexual men in $30 \%$ to $70 \%$ of patients, ${ }^{1-3}$ but is less common in homosexual men. ${ }^{7}$ PGU may be associated with symptoms such as urethral discharge and/or dysuria, or may be asymptomatic when the diagnosis is based solely on the finding of excess polymorphonuclear leucocytes on a gram-stained urethral smear.

In the majority of studies the condition has been most commonly associated with the isolation of the

Department of Genitourinary Medicine and Medical Microbiology, West London

B S Azadian

Charing Cross Hospital, London, UK

K A McLean, B A Evans, J M H Lim genital serotypes of Chlamydia trachomatis from the urethra, presumably due to concomitant chlamydial infection. ${ }^{6}$ Ridgway and Oriel have recommended the addition of a course of tetracycline to routine single dose treatment of gonorrhoea. ${ }^{7}$ The aetiology of PGU in chlamydia-negative cases has not been established. One possible contender, Ureaplasma urealyticum, was not found to be associated with PGU in two studies. ${ }^{28}$

The aims of this study were to identify organisms which might be associated with the occurrence of PGU, and to observe the effect of doxycycline prescribed as a seven day course at the time of diagnosis and single dose treatment of urethral gonorrhoea in heterosexual men.

\section{Patients and methods}

STUDY POPULATION

Consecutive heterosexual men attending the Department of Genitourinary Medicine at West London Hospital for diagnosis and treatment of acute urethritis were invited to participate in the study and given an explanatory leaflet. Patients were selected by a provisional diagnosis of gonococcal urethritis on the basis of characteristic intracellular Gram-negative diplococci in a urethral smear, later confirmed by isolation of Neisseria gonorrhoeae in culture. Patients were excluded from the study if they had been taking antibiotics during the previous two weeks, if they were unlikely to reattend for follow-up tests, or it they were allergic to tetracycline.

At the initial visit urethral specimens were taken for microscopic examination and culture for $N$ gonorrhoeae, $C$ trachomatis, Mycoplasma hominis, $U$ urealyticum and Gardnerella vaginalis. Blood was taken for serological tests for syphilis.

PGU was diagnosed at first follow-up (day 14) if ten or more polymorphonuclear leucocytes were seen per high power field ( $x 100$ objective; average number of polymorphs seen in five fields) on microscopic examination of the Gram-stained urethral smear.

\section{ISOLATION OF NEISSERIA GONORRHOEAE}

Specimens were taken with a plastic loop for microscopic examination and culture for $N$ gonorrhoeae. Specimens were plated directly on to Modified New 
York City Medium (Oxoid) and cultured for 48 hours in a candle extinction jar. $N$ gonorrhoeae were identified by colonial appearance, oxidase reaction, fluorescent antibody test (Difco) and carbohydrate utilisation tests when necessary. Penicillinaseproducing $N$ gonorrhoeae (PPNG) were detected using a chromogenic cephalosporin test (Nitrocefin, Oxoid Ltd.)

\section{ISOLATION OF CHLAMYDIA TRACHOMATIS}

A wire-mounted, cotton wool tipped swab was used to take a urethral specimen. The swab was placed in transport medium and stored at $-70^{\circ} \mathrm{C}$ until it was inoculated on to $\mathrm{McCoy}$ cells. The preparation was centrifuged at $3000 \mathrm{~g}$ for one hour at $35^{\circ} \mathrm{C}$ and the cells were treated with cycloheximide, incubated at $37^{\circ} \mathrm{C}$ for 48 hours, fixed in methanol and stained with iodine. Microscopic examination for the characteristic inclusions was performed.

\section{ISOLATION OF MYCOPLASMA HOMINIS AND UREAPLASMA UREALYTICUM}

Mycoplasma agar base (Oxoid) and PPLO broth base (Difco) were supplemented by the addition of $10 \%$ yeast extract, $20 \%$ horse serum and 1000 I.U. penicillin per $\mathrm{ml}$. The broth was further supplemented with either $0.2 \%$ arginine and $0.001 \%$ phenol red (arginine broth, $\mathrm{pH} 7.4$ ) or $0.1 \%$ urea, $0.001 \%$ phenol red and $15 \mu \mathrm{g} / \mathrm{ml}$ lincomycin (ureaplasma broth, $\mathrm{pH} 6.5)$. Thallous acetate $(0.25 \%)$ was added only to the mycoplasma agar. Two urethral specimens were obtained using wire-mounted nasopharyngeal (calcium alginate) swabs. One swab was placed in transport medium and delivered to the laboratory within two hours. The swab was then plated on to mycoplasma agar before being expressed into arginine broth. Both cultures were incubated at $37^{\circ} \mathrm{C}$ for seven days, the agar in $5 \% \mathrm{CO}_{2}$ and the broth aerobically. The broth was subcultured on to mycoplasma agar if there was a colour change from orange to magenta. Typical "fried egg" colonies on the mycoplasma agar were regarded as positive for $M$ hominis.

The second swab was expressed directly into ureaplasma broth and serial ten-fold dilutions made up to $1: 1000$. These were incubated aerobically at $37^{\circ} \mathrm{C}$ and examined over 72 hours for colour change from yellow to magenta. The highest dilution showing a colour change was subcultured on to blood agar to exclude contaminants. In the absence of contaminants such cultures were recorded as positive for $U$ urealyticum.

\section{ISOLATION OF GARDNERELLA VAGINALIS}

Specimens were inoculated on to Human Blood Agar bi-layer plates with antibiotic selective supplement (gentamicin sulphate $4 \mathrm{mg} / 1$ medium, nalidixic acid $30 \mathrm{mg} / 1$ medium, amphotericin B $2 \mathrm{mg} / 1$; Oxoid Ltd.,
Basingstoke, England) and incubated in an $8 \% \mathrm{CO}_{2}$ atmosphere for 48 hours. Colonies that consisted of Gram-variable bacilli, showing beta-haemolysis on human but not horse blood agar, were catalasenegative, susceptible to metronidazole $50 \mu \mathrm{g}$ disc (Oxoid) and resistant to sulphonamide $1,000 \mu \mathrm{g}$ disc (Oxoid) were identified as $G$ vaginalis.

\section{TREATMENT AND FOLLOW UP}

Patients were given single dose treatment with oral ampicillin $3 \mathrm{~g}$ plus probenecid $1 \mathrm{~g}$. Alternatively intramuscular spectinomycin $2 \mathrm{~g}$ was given if the patient was allergic to penicillin or infection with penicillinase-producing Neisseria gonorrhoeae (PPNG) was suspected on the basis of a history of sexual contact in an area with a high prevalence of PPNG infection, in particular the Far East or Central Africa. In addition patients were randomised by a computer generated code to receive either oral doxycycline $100 \mathrm{mg}$ twice daily for seven days or one placebo capsule twice daily for seven days. This protocol was approved by the District Ethical Subcommittee.

Patients were asked to reattend for follow-up on the 14th day after initial presentation. Urethral specimens were taken for microscopy and for repeat cultures of $N$ gonorrhoeae, $C$ trachomatis, $U$ urealyticum, $M$ hominis and $G$ vaginalis. A second follow-up was requested on day 28 when further urethral specimens were taken for microscopy and culture of $N$ gonorrhoeae and serological tests for syphilis were repeated.

\section{STATISTICAL ANALYSIS}

Statistical correlates were calculated using the chisquare test with Yates's correction.

\section{Results}

Three hundred and thirty five patients were enrolled into the study. One hundred and nineteen of these were excluded from analysis because they failed to attend for initial follow-up tests (87), they failed to take the course of doxycycline or placebo capsules (11), or they were unable to be assessed for PGU because of persistence of gonococcal infection due to reinfection or treatment failure (21). Therefore 216 men were assessable for the presence of PGU at initial follow-up, 104 treated with placebo and 112 with doxycycline.

Of the 335 gonococcal isolates detected during the study period, $18(5.4 \%)$ were PPNG, six in the placebo group and 12 in the doxycycline treatment group. Nine chlamydia results were not available owing to technical problems and in the remaining 326 cases $C$ trachomatis was isolated in culture in $\mathbf{4 6}$ $(14.1 \%), 20$ in the placebo group and 26 in the doxycycline treatment group. There were no sig- 
Table 1 Occurrence of PGU

\begin{tabular}{|c|c|c|c|}
\hline & Placebo & Doxycycline & Total \\
\hline PGU (> 10 PMNL/hpf) (\%) & $\begin{array}{r}54 / 104(51 \cdot 9) \\
\chi^{2} y=\end{array}$ & $\begin{array}{l}30 / 112(26.8) \\
3.30 ; \quad \text { p }<\end{array}$ & $\begin{array}{l}84 / 216 \\
(38 \cdot 9) \\
001\end{array}$ \\
\hline Symptomatic PGU (\%) & $\begin{array}{r}7 / 104(6 \cdot 7) \\
\chi^{2} y=1\end{array}$ & $\begin{array}{ll}3 / 112 & (2 \cdot 7) \\
12 ; & 0.2<\end{array}$ & $\begin{array}{l}10 / 216 \\
(4.6) \\
<0.3\end{array}$ \\
\hline
\end{tabular}

$\chi^{2} y=$ Chi square with Yates's correction

PMNL = Polymorphonuclear leucocytes

nificant differences in the initial isolation rates of PPNG nor $C$ trachomatis in the doxycycline and placebo groups ( $p>0.05$ for both).

Of the 216 patients suitable for assessment at first follow-up, 84 (38.9\%) were diagnosed by microscopy as having PGU, 54/104 (51.9\%) in the placebo group and $30 / 112(26.8 \%)$ in the doxycycline group (table 1). This difference was highly significant $(p<$ 0.001 ). However, PGU associated with symptoms such as urethral discharge or dysuria was uncommon, occurring in $7 / 104(6.7 \%)$ of the placebo group and $3 / 112(2 \cdot 7 \%)$ of the doxycycline group.

Of the 216 assessable patients, side effects related to treatment were uncommon, occurring in seven patients in the placebo group and nine in the doxycycline group. In the majority of cases side effects occurred within 24 hours of single dose ampicillin and were of less than $\mathbf{4 8}$ hours duration. One patient complained of nausea after spectinomycin. However, four of the nine patients in the doxycycline group stopped taking the capsules because of vomiting (2), nausea (1) or headache (1), while one patient discontinued placebo because of nausea.

ISOLATION OF CHLAMYDIA AND DEVELOPMENT OF PGU (table 2)

After exclusion of five patients in whom chlamydial isolation was not assessable owing to technical reasons, isolation of $C$ trachomatis on entry in the placebo group was associated with the occurrence of PGU in $12.1 \%$ (12/99) of cases. Seventy one \% (12/ 17) of patients in whom $C$ trachomatis was isolated on entry developed PGU. However, this association did not reach significance.

In the placebo group persistence of chlamydial infection at first follow-up was associated with the development of PGU in $8 / 90(8.9 \%)$ patients, while one patient with persistence of the organism did not develop PGU. Therefore PGU developed in 8/9 $(89 \%)$ patients with persistence of chlamydial infection. This association was significant $(p<0.05)$.

As expected, patients given doxycycline were less likely to develop PGU. Of 21 patients in whom $C$ trachomatis was isolated on entry, six developed PGU, while persistence of the organism occurred in only one patient. (Data not shown in table).

ISOLATION OF M HOMINIS, U UREALYTICUM AND $G$ VAGINALIS (table 2)

One hundred patients in the placebo group had initial cultures for $M$ hominis, $U$ urealyticum and $G$ vaginalis and attended for follow-up. Ninety of these patients had second cultures for these organisms.

Of 12 patients in whom $M$ hominis was isolated on entry, 10 developed PGU, suggesting an association between the isolation of this organism and the occurrence of PGU. However, the association did not reach significance $(0 \cdot 1>p>0.05)$. Three of these 12 patients were also positive for $C$ trachomatis on entry, and exclusion of these three reduced the association with PGU further $(0.3>p>0.2)$.

Of five patients in whom $M$ hominis was isolated on entry and at day 14, four developed PGU. However, this association was not significant.

$U$ urealyticum was isolated on entry in $38 / 100$ patients in the placebo group and 22 of these 38 patients developed PGU. There was no association between isolation of this organism and development of PGU ( $p>0.6)$.

Persistence of $U$ urealyticum at day 14 occurred in $23 / 90$ patients in the placebo group and was not

Table 2 Isolation of $C$ trachomatis, $M$ hominis and $U$ urealyticum related to $P G U$ in placebo group

\begin{tabular}{|c|c|c|c|c|c|}
\hline Organism & & $P G U$ & No PGU & $\chi^{2} y$ & pvalue \\
\hline C trachomatis day 0 & $\begin{array}{l}+ \\
-\end{array}$ & $\begin{array}{l}12 \\
40\end{array}$ & $\begin{array}{r}5 \\
42\end{array}$ & $1 \cdot 88$ & $0.2>p>0.1$ \\
\hline$C$ trachomatis days 0 and & + & $\begin{array}{r}8 \\
38\end{array}$ & 43 & $4 \cdot 16$ & $0.05>p>0.02$ \\
\hline$M$ hominis day 0 & $\begin{array}{l}+ \\
-\end{array}$ & $\begin{array}{l}10 \\
44\end{array}$ & $\begin{array}{r}2 \\
44\end{array}$ & 3.48 & $0.1>p>0.05$ \\
\hline$M$ hominis days 0 and 14 & \pm & 44 & 41 & 0.59 & $0.5>p>0.4$ \\
\hline$U$ urealyticum day 0 & + & $\begin{array}{l}22 \\
32\end{array}$ & $\begin{array}{l}16 \\
30\end{array}$ & $0 \cdot 16$ & $0.7>p>0.6$ \\
\hline U urealyticum days 0 and & $\stackrel{+}{-}$ & $\begin{array}{l}13 \\
35\end{array}$ & $\begin{array}{l}10 \\
32\end{array}$ & $0 \cdot 01$ & $p>0.9$ \\
\hline
\end{tabular}


associated with the development of PGU ( $p>0.9$ ).

In the doxycycline group there was no association between the development of PGU and isolation of $M$ hominis on entry ( $p>0.5$ ), persistence of $M$ hominis at day $14(\mathrm{p}>0.5)$, isolation of $U$ urealyticum on entry ( $p>0.5)$ nor persistence of $U$ urealyticum at day $14(p>0.5)$. (Data not shown in table).

Of 103 patients treated with doxycycline, $13 \mathrm{had}$ persistence of $U$ urealyticum at day 14.

$G$ vaginalis was isolated on entry in only one patient in the study.

\section{Discussion}

The incidence of PGU in the placebo group was $52 \%$, which falls within the range of 30 to $70 \%$ reported in previous studies. ${ }^{1-3}$ PGU was associated with symptoms in only $13 \%(7 / 54)$ of cases in this group. Failure to perform a urethral smear at day 14 and chlamydial testing on presentation would have resulted in 47 of $54(87 \%)$ patients with microscopic PGU being untreated due to the absence of symptoms. Forty seven of $104(45 \%)$ patients with gonorrhoea in the placebo group had asymptomatic PGU diagnosed at day 14. Therefore PGU will pass undiagnosed unless it is carefully sought, particularly in departments without adequate chlamydial diagnostic facilities.

A course of doxycycline $100 \mathrm{mg}$ taken twice daily for one week was associated with a highly significant reduction $(\mathrm{p}<0.001)$ in the incidence of PGU $(51.9 \%$ in the placebo group vs $26.8 \%$ in the doxycycline group). This supports the findings of Ridgway and Oriel who compared the effect of adding a course of oxytetracycline $500 \mathrm{mg}$ orally four times daily for 7 days to single dose oral treatment for gonorrhoea with a historical group treated with ampicillin and probenecid alone. ${ }^{7}$ In their study PGU was diagnosed in nine $(20 \%)$ of 45 patients treated with oxytetracycline in addition to ampicillin and probenecid. In our study $30(27 \%)$ of 112 patients treated with doxycycline in addition to single dose treatment for gonorrhoea developed PGU. Therefore although the addition of doxycycline resulted in a significant reduction in the development of PGU, there remained a considerable proportion of patients who still developed PGU. In both studies the addition of a tetracycline resulted in almost complete elimination of $C$ trachomatis at follow-up in patients positive on entry, so it would appear likely that PGU in this group is due to a cause other than $C$ trachomatis.

The chlamydial isolation rate of $14 \%$ on entry to the study was lower than we had expected, but fell within the range of 11 to $34 \%$ reported in earlier studies. ${ }^{59}$ Similarly the chlamydial isolation rate of $23 \%(12 / 52)$ on entry in patients in the placebo group who went on to develop PGU was lower than in some studies but within the reported range of 15 to $81 \% .^{15}$

Isolation of $M$ hominis on entry to the study was associated with the development of PGU in $83 \%$ (10/ 12) of patients in the placebo group, but the association was not significant, and was reduced further after exclusion of three patients in whom $C$ trachomatis was also isolated. Persistence of $M$ hominis at follow-up was associated with PGU in $80 \%(4 / 5)$ of cases, but the small numbers involved were not compatible with the demonstration of a significant difference.

The addition of doxycycline was associated with a lower incidence of PGU $(3 / 13,23 \%)$ in patients with $M$ hominis and persistence of the organism occurred in only two cases, neither of whom developed PGU.

These figures suggest an association of $M$ hominis with PGU but statistical significance could not be shown. A larger group of patients would have to be studied in order to establish whether the association would reach significance.

Neither the initial isolation of $U$ urealyticum nor the persistence of the organism was associated with the development of PGU. This finding is in accordance with earlier studies. ${ }^{2} G$ vaginalis was isolated in only one patient in the study.

We did not routinely examine urethral specimens for the presence of Trichomonas vaginalis as previous work has shown that this organism is rarely isolated in these patients. ${ }^{8}$

This study confirms that a significant reduction in the incidence of PGU may be achieved by the addition of a course of tetracycline to standard single dose treatment for urethral gonorrhoea. There remains a considerable proportion of patients in whom the aetiology of PGU is unknown.

Address for reprints: Dr K A McLean, Department of Genitourinary Medicine, West London Hospital, Hammersmith Road, London W6 7DP, UK

1 Richmond SJ, Hilton AL, Clarke SKR. Chlamydial infection: Role of Chlamydia Subgroup $A$ in non-gonococcal and postgonococcal urethritis. Br J Venereal Dis 1972;48:437-44.

2 Vaughan-Jackson JD, Dunlop EMC, Darougar S, Treharne JD, Taylor-Robinson D. "Urethritis due to Chlamydia trachomatis." Br J Venereal Dis 1977;53:180-3.

3 Terho P. "Chlamydia trachomatis in gonococcal and postgonococcal urethritis." Br J Venereal Dis 1978;54:326-9.

4 Bowie WR, Alexander ER, Holmes KK. Etiologies of postgonococcal urethritis in homosexual and heterosexual men: roles of Chlamydia trachomatis and Ureaplasma urealyticum. Sex Transm Dis 1978;5:151-4.

5 Alani MD, Darougar S, MacDonald Burns DC, Thin RN, Dunn $H$. "Isolation of Chlamydia trachomatis from the male urethra". Br J Venereal Dis 1977;53:88-92.

6 Perroud HM, Miedzybrodzka K. "Chlamydial infection of the urethra in men." $\mathrm{Br} J$ Venereal Dis 1978;54:45-9.

7 Ridgway GL, Oriel JD. "Advantages of adding a course of tetracycline to single dose ampicillin and probenecid in the treatment of gonorrhoea." Br J Venereal Dis 1984;60:235-7.

8 Holmes KK, Handsfield HH, Wang SP, Wentworth BB, Turck M, Anderson JB, Alexander ER. Etiology of nongonococcal urethritis. N Engl J Med 1975;292:1199-205.

9 Oriel JD, Reeve P, Thomas BJ, Nicol CS. "Infection with Chlamydia Group A in men with urethritis due to Neisseria gonorrhoeae." J Inf Dis 1975;131:376-82:

Accepted for publication 12 October 1989 